\title{
Mother-Child Emotional Availability Mediating the Effects of Maternal Psychological Well-being and Child's Cognitive Competence on Child Behavior Problems
}

Recent intervention studies document that mother-child dyads with higher levels of Emotional Availability (EA) report fewer child behavior problems than dyads with lower EA. This study examines possible mechanisms that lead to this result by looking at the parent-child micro-system as a whole, with multi-dimensional relationships that include individual differences in the child's cognitive level, parental stress and parent-child interaction. A total 67 children (11/2 to $51 / 2$ years of age) and their mothers were videotaped during 30-min play interactions. Interactions were coded using the Emotional Availability (EA) Scales (Biringen, Robinson, \& Emde, 1998). Mothers completed Parenting Stress Index-Short Form, Child Behavior Checklist/1 1/2 - 5, and the Ages Stages Questionnaire. The findings showed that mothers with higher levels of parenting stress were more likely to be intrusive, hostile, insensitive, and had a tendency to do less structuring in play. The children of stressed and depressed mothers demonstrated less involvement and responsiveness towards their mothers. Children who have higher dyadic EA scores experienced fewer externalizing and internalizing problems. SEM analyses results showed a mediation effect of EA on the association between maternal psychological well-being and child behavior problems. Fewer deficits in child communication skills and problem solving skills that were related with lower parenting stress and depression were associated with higher maternal nonintrusiveness. Higher non-intrusiveness was related to less internalizing and externalizing problems that indicated the

Assistant Professor, Dept. of Child and Family Studies, Yonsei University (mjkang@yonsei.ac.kr)

Key Words: Emotional availability, cognitive competence, behavior problems, maternal psychological well-being indirect effect of child cognitive competence. Possible interpretations and implications of the study findings are discussed.

Recent intervention studies report that improvement of the mother-child Emotional Availability (EA) or the quality in the dyadic parent-child interaction correlates to decreased child behavior problems (Dombrowski, Timmer, Blacker, \& Urquiza, 2005; van Doesum, Riksen-Walraven, Hosman, \& Hoefnagels, 2008). This study explores and examines the mechanism that explains this relationship. Maternal psychological well-being such as parental stress or depression is known to influence low maternal sensitivity; in addition, low emotional availability is known to negatively influence child outcome. However, limited studies examine the whole mechanism in consideration of the fact that individual differences in a child's cognitive level may also affect parental stress and parent-child interaction. This study explores the direct and indirect effect of maternal psychological well-being, motherchild EA, and child cognitive functioning when predicting child behavior problems.

Human behavior and competencies are often the result of interactions between emotional, social and cognitive variables over the course of a lifetime that are both causes and consequences of our relationships (Bandura, 1989). During the early years of development, many of our most important activities occur in dyadic relationships with primary 
caregivers who act as mentors (Vygotsky, 1978), guardians of our well being (Bronfenbrenner, 1979), and social role models (Bandura, 1989). The interactions are often impacted by the social ties between participants and especially the capabilities of the stronger members of the dyadic interaction in structuring and guiding activities. One variable that may set the tone for mother-child activities is the quality of the directed activity itself. And one of the major constructs that determine the quality of the interaction is the EA of child and the primary caregiver.

Studies on maternal psychological well being suggests that maternal depression functions as one of the major risk factors for the development of behavioral problems (Alpern \& Lyons-Ruth, 1993; Downey \& Coyne, 1990; Leadbeater, Bishop, \& Raver, 1996). Other studies indicate that negative maternal parenting behaviors are often interconnected with maternal depression that serve as predictors of the child's maladjustment (externalizing and internalizing behaviors). For example, Harnish, Dodge, and Valente's (1995) study showed that maternal self-report depressive symptoms and negative mother-child interaction quality were significant predictors of children's externalizing behaviors.

The literature supports the idea that maternal psychological well-being (A) is related to the maternal interactive behavior (B) and child behavior problems (C); in addition, the maternal interactive behavior (B) is related with child behavior problems (C). Caregiver-research shows a pair-by-pair relation between the constructs $(\mathrm{A} \rightarrow \mathrm{B}, \mathrm{B} \rightarrow \mathrm{C}$, and $\mathrm{A} \rightarrow$ C). Therefore, it can be expected that the $\mathrm{A} \mathrm{C}$ relations may be accounted for by the level of $\mathrm{B}$. Unfortunately, limited studies have directly studied this possible phenomenon. Previous studies in this area have focused on maternal behavioral factors that are different from what EA Scales assess. The EA emphasizes mutually negotiated dyadic interaction.

The Emotional Availability Scales (EAS; Biringen, Robinson, \& Emde, 1998) is an observational methodology designed to assess parent-child interaction during infancy and beyond. Inspired by Ainsworth's Sensitivity-Insensitivity Scale (Ainsworth,
Blehar, Waters, \& Wall, 1978) and Mahler, Pine and Bergman's (1975) concept of Emotional Refueling, Biringen and her colleagues incorporated different aspects of caregiver-child relationships into their EAS that enabled the direct assessment of mutual responsiveness and enjoyment in dyadic interaction within various settings and age groups (Biringen, Robinson, \& Emde, 2000; Bretherton, 2000). The EA Scales consist of six dimensions: four EA qualities measure the adult's side and two EA qualities measure the child's side toward the adult. The adult dimensions are sensitivity, structuring, non-intrusiveness, and non-hostility; the child dimensions are responsiveness to the adult and involvement of the adult. The scoring system of EA includes the role of child in the interaction with the mother. The mother's EA scores cannot be assessed without consideration of the child's responses and their emotional status. In order to be considered as a sensitive and well structuring mother, her attempts to be sensitive or to provide optimal structuring should be accepted by her child. The key element is emotional attunement, matched emotional status, and genuineness within the dyads. The underlying concept of maternal sensitivity is similar to Ainsworth's sensitivity scale in that both the parent and child scales are highly global assessments of the interaction and focus on the general behavior styles rather than discrete behaviors (Biringen, Robinson, \& Emde, 2000). Some researchers have summarized major differences between the EA Scales and the Ainsworth's maternal sensitivity scales (Bretherton, 2000; Emde, 2000): 1) The EA focus more explicitly on emotion through the emphasis of a parent's EA and affect attunement. 2) The EA focus on both partners EA to each other for the assessment of mutual negotiation and contributions. 3) The EA allows the assessment of the quality of parent-child interaction in all age groups whereas Ainsworth maternal sensitivity scale is more adequate for assessing mothers with infants.

A purpose of this study was to examine how the well-being of the mother may be connected with mother's and child's EA in their dyadic relationship. Previous EA research has uncovered that mothers who had children with less secure attachment at 18 
months and who showed more depressive symptoms when the children were age 7 showed less optimal mother-child EA at age 7 (Easterbrooks, Biesecker, \& Lyons-Ruth, 2000). This study showed that maternal depression affects the interactions between mother and child; in addition, it studied the mechanisms by which maternal psychological well-being can affect mother-child EA and/or children's outcomes is still left as a question to be explained. Previous studies focused on the mother's parenting behavior or maternal sensitivity; however, a question can be raised about the role of the child's EA and individual characteristics in determining the level of parenting stress and the quality of mother-child interaction.

The second purpose of this paper was to examine the effects of the children's developmental status (level of cognitive functioning) on maternal psychological well-being and mother-child EA and to examine the mechanism of those three constructs when predicting child behavior problems. According to Crowell and Feldman (1988), a child's delay in development often causes the mother to be a more "worried-mother" about completing a task and to be more controlling of a child's appropriate behaviors. Is can be assumed that EA is a factor that predicts child's behavior problems along with the child's own resources and characteristics that may serve as a contributing factor to a child's behavior problems. In this vein, Moreno, Klute, and Robinson (2007) reported that child's cognitive and social resources mediate the relationship between maternal EA and child's empathy toward both mother and stranger (this study assessed only maternal EA and excluded child EA). If the child has a high level of communicative competence and problem solving skills, such skills can function as protective factors to help the depressed or stressed mother communicate with the child and the feedback loop was observed during actual interaction in our study. In contrast, if the child has any sort of disability (hearing or speaking) then the child may require extra parental skills.

Maternal depression is associated with the quality of mother-child interaction and this quality is associated with child behavior problems, the first hypothesis was that EA will account for the association between maternal psychological well- being and child behavior problems. The second hypothesis was that deficits in child cognitive competence would have a negative effect on the maternal psychological well-being and EA, which would eventually have an effect on child behavior problems. This assumption implies the indirect effect of child's cognitive competence on child behavior problem. The children's competence in communication and problem solving skills are considered to enhance or interfere with social interaction skills. These two major hypotheses are based on the assumption that both the mother's and child's difficulties will have an indirect effect on child's behavioral outcomes.

Limited studies have examined inter-related variables that stem from activities in the parent-child micro-system such as, individual differences in the child's cognitive level, parental psychological wellbeing, and parent-child interaction that use multidimensional relations. The idea that individual indicators such as the mother's well-being and cognitive development of children may be interrelated is critical to understand the importance of EA and the degree to which intervention based on EA may have significant impact on the life trajectories of mother and child.

\section{METHODS}

\section{Participants}

The sample consisted of 67 children (44 boys, 23 girls), ranging in age from $1 \frac{1 / 2}{2}$ to $5 \frac{1 / 2}{2}$-years of age (Mean age for children: 46.3 months, $\mathrm{SD}=11.8$; Mean age for mothers: 30.6 years, range: 20-62 years. $\mathrm{SD}=8.63$ ). Yearly household income information was garnered in a categorical manner and was later coded into an ordinal scale. Thirty-seven families (55.2\%) reported their household income under $\$ 25,000,15$ families (22.4\%) reported between $\$ 25,000-\$ 49,999,13$ families (19.4\%) between $\$ 50,000$ $\$ 99,999$, and 2 families (3\%) above $\$ 100,000$. Children were referred for outpatient services due to externalizing behavior problems. Approximately half the mothers $(n=34)$ had a high school diploma or less: 21 mothers (31.3\%) had earned a high school 
diploma, while 13 of the mothers (19.4\%) had not graduated from high school. The remaining 33 mothers, 16 (23.9\%) had completed some college education, 7 (10.5\%) had earned an associate's degree or had attended some vocational school, and 10 (14.9\%) had a bachelor's degree. Most of the families were Caucasian ( $\mathrm{n}=53$ or $79.1 \%)$, nine families were African-American (13.4\%), four families were Latino (6\%), and one was Asian (1.5\%). The data were collected at a local children's hospital in the Midwest U.S.

\section{Procedure}

The subjects were recruited through a modified snowball sampling. The researcher contacted the pediatrician and the program coordinator working at the children's hospital and discussed about the study. Families who visited the children's hospital to consult on a child's behavior problems were introduced to the study by the program coordinator at the hospital. Some other families that visited the children's hospital were approached by the examiner and asked if they would be interested in participating. The mothers and the children were briefly notified about the present study and only those who agreed to participate were included in the study. Participants were nominally reimbursed for the time needed to participate in the study. For the research ethics, the researcher who videotaped and scored the interaction was trained through "Children's HIPAA Program" the federal Health Insurance Portability and Accountability Act and was approved to conduct this research by the Vice President of Information Services and the President/ CEO of the children's hospital. The examiner signed the form of agreement about the privacy protection policy that indicated compliance with federal law.

Assessment protocols, procedures, and tools were the same for all 67 dyads. Mothers and children were videotaped at not home or in a quiet playroom at the children's hospital, with the setting chosen by the participants based on convenience. The toys and activities provided were the same for both settings. Mean values for the dependent variables did not vary by setting (home versus playroom). Motherchild dyads were asked to play together for approximately $30 \mathrm{~min}$, and all interaction was videotaped. The session consisted of structured activities, followed by unstructured play. The structured activities included 5-min block design and 5-min puzzle solving. The levels of difficulty for the structured tasks were adjusted to fit the child's age. For the block design, children three years of age or less were provided with a shape sorter (inserting the star-shaped block piece into a star-shaped hole, and the same for the circle, square, and diamondshaped puzzles). For children older than three, a cardboard design and a set of blocks with the same shapes and colors were provided. The task was to make a block design that matched the cardboard picture (a rocket or a house made of seven to eight pieces). The examiner explained to the mother and the child that they could work together and that the blocks should be next to or in front of the cardboard (but not on top of it). The examiner gave notice at the end of five min. The next structured task was to complete a puzzle. For the children three years of age or less, puzzles with five pieces (slice of pizza, apple, boat, goose, and banana) were given to place into the matching spot. Children older than three were provided with more complicated puzzles (a picture of a house with a garden and clouds). The examiner gave notice at the end of five min; however, with the offer of some additional time if they wanted to complete the puzzle. Following these structured episodes, mother-child interaction was observed for a 20-min period of unstructured play (with no specific goal of completing a task). Developmentally appropriate Lego toys were provided. Children three and younger were given a farm set, while older children were given a car set with a road for driving. After the play session, mothers were asked to complete the Child Behavior Checklist (CBCL), a self-report of depressive symptoms (CES-D), the Parenting Stress Index-Short Form (PSI-SF), and the Ages Stages Questionnaire (ASQ).

\section{Measurements}

EA. Infancy to Early Childhood Version of the EA (EA) Scales ( $3^{\text {rd }}$ ed.; Biringen, Robinson, \& Emde, 1998) was used to assess the quality of the interaction between the mother and child. The EA Scales include 
four dimensions to describe adult behavior towards the child in the course of dyadic interaction (i.e., sensitivity, structuring, non-intrusiveness, and nonhostility) and two scales evaluating the child's behavior towards the adult during the interaction (responsiveness and involvement).

Three raters were trained for three months by viewing the Biringen's training tapes, reading the manual, and attending regular meetings. At the end of the training, the raters independently viewed the 8 episodes provided in Biringen's training tapes and rated the EA Scales. The scores of the 8 episodes rated by the three raters were sent to the first author of the EA Scales via email and were approved as reliable in inter-lab reliability (inter-rater reliability was examined before the actual assessment). Thirty cases were chosen from the data and randomly assigned to two raters. The scales were coded after viewing each episode as a whole (30 $\mathrm{min})$. The intraclass correlation coefficient (ICC; Shrout \& Fleiss, 1979) ranged from .91 to .97 (maternal sensitivity scale that ranges from 1 to 9 made it harder to get the exact same scores between the raters compared to other scales with smaller scoring ranges). The trained coders were blind to all information about the subjects (except for age and gender) and independently scored the EA Scales.

\section{Child Behavior Problems The Child Behavior} Checklist (CBCL/1 1/2 - 5, Preschool Form; Achenbach \& Rescorla, 2000) was used to rate behavior problems in children. The CBCL/1 $1 / 2-5$ asks parents to rate 99 items, and provides three summary measures: externalizing problems, internalizing problems, and total problems. Internal consistency reliability (Cronbach's alpha) was .89 for the internalizing and .92 for the externalizing subscales.

Parenting Stress The 36-item Parenting Stress Index-Short Form (PSI-SF; Abidin, 1995) was used. The PSI-SF measures three types of parenting stress. "Parental Distress" refers to the stresses attributed to the parenting role, including a sense of competence, restrictions imposed on life by the demands of parenting, inter-parental conflict, lack of social support, and depression. "Parent-Child Dysfunctional
Interaction" refers to the parent's perception that the child is not meeting the parent's expectations and that interactions with the child are not satisfying or reinforcing to the parent. Finally, "Difficult child" refers to the behavioral characteristics of the child that make the child difficult to manage. The PSI-SF also yields a "Total stress score." The Cronbach's alpha reliability for the PSI-SF total score was .95.

Maternal Depression The Center for Epidemiological Studies-Depression Scale (CES-D; Radloff, 1977), a 20-item self-report questionnaire, was used to assess current symptoms of maternal depression. The CES-D identifies the presence and severity (number of symptoms weighed by frequency/ duration) of depressive symptoms. The possible range of scores is 0-60, with higher scores indicative of greater depressive symptomatology. Cronbach's alpha for the CES-D total score was .85 .

Child Cognitive Competence The Ages and Stages Questionnaires (ASQ, 2 ${ }^{\text {nd }}$ Ed.; Squires, Potter, \& Bricker, 1999) for the $16,20,33,36,42,48,54$, and 60 months was completed by the mother as a screener for the cognitive developmental status of the children and used the appropriate form for the child's age. The questionnaire contains 30 developmental items, divided into five specific developmental areas. In the present study, Communication and the Problem Solving scales were used as measures of child's cognitive competence. Communication addresses a child's babbling, vocalizing, listening, and understanding. The Problem Solving addresses a child's ability to learn and play. For each item, mothers checked "yes" (scored 10 points) to indicate that their child performs the behavior specified in the item, "sometimes" (scored 5 points) to indicate an occasional or emerging response from their child, or "not yet" (scored 0 points) to indicate that their child does not yet perform the behavior. The summed scores for each area are then compared with empirically derived cut-off points. The ASQ proportional scores are the cut-off scores divided by the child's actual score in the domain. A proportional score of 1 or greater indicates that the child's level might be below the average develop- 
mental age level or that they have a deficit in that area; therefore, higher ASQ scores indicate lower cognitive competence.

\section{RESULTS}

\section{Demographic Characteristics and Study Variables}

The demographic variables collected in a form of ordinal or ratio scale (age, education, and family income) were analyzed through zero-correlation to see its relation with the study variables. For the variables collected as a nominal scale (e.g., gender), the mean differences of the study variables were tested through using independent $t$-tests. None of the demographic variables listed were correlated with the EA scales or a child's cognitive functioning; however, mother's education level and household income both were negatively related with maternal depression ( $r=-.30, r=-.29, p s=.01$, respectively). Only one mean difference by gender was found as significant. The mothers of boys $(\mathrm{M}=3.93, \mathrm{SD}=.73)$ were rated higher in maternal structuring than the mothers of girls $(\mathrm{M}=3.57, \mathrm{SD}=.59 ; t(65)=2.08$, $p=.04$. The descriptive statistics of the study variables are depicted in Table 1 . Since the effect of the gender, age, education, and income was systematically unrelated to the study variables, the demographic variables were excluded in the SEM models. The skewness of the study variables were mostly below 1 with only two exceptions (maternal depression=1.01 and child internalizing=1.14). However, these values were less than 2 , so the normality of the distribution was not considered problematic.

\section{Relations among Study Variables}

Prior to running the SEM, correlations among the major study constructs (EA Scales, maternal depression, parenting stress, child cognitive competence, and internalizing and externalizing problems) were examined using SPSS 18.0 for Windows (Table 2). In general, EA Scales correlated negatively with internalizing and externalizing scores. Maternal depression and parenting stress was negatively correlated with the EA Scales, with both the maternal and the child's side of EA. That is, the dyads reported to have higher parenting stress and depression scores were observed as having lower mother-child EA. Interestingly, maternal qualities such as non-intrusiveness and non-hostility were significantly linked with parenting stress and depression (although the EA literature rarely

Table 1. Descriptive Statistics of the Study Variables $(n=67)$

\begin{tabular}{|c|c|c|c|c|c|}
\hline Construct & Variables & Mean & $\mathrm{SD}$ & Min. & Max. \\
\hline \multirow{6}{*}{ EA } & M-Sensitivity (1-9) & 6.33 & 1.83 & 3.0 & 9.0 \\
\hline & M-Structuring (1-5) & 3.81 & 0.70 & 2.0 & 5.0 \\
\hline & M-Non-intrusiveness (1-5) & 3.93 & 0.96 & 2.0 & 5.0 \\
\hline & M-Non-hostility (1-5) & 4.48 & 0.70 & 3.0 & 5.0 \\
\hline & C-Involvement (1-7) & 5.24 & 1.53 & 2.0 & 7.0 \\
\hline & C-Responsiveness (1-7) & 5.15 & 1.54 & 2.0 & 7.0 \\
\hline CES-D & M-Depression total & 15.25 & 10.88 & 1.0 & 45.0 \\
\hline Parenting Stress & M-Parental stress total & 84.40 & 22.88 & 49 & 131 \\
\hline \multirow{3}{*}{ Behavior Problems } & C-Internalizing & 53.82 & 12.42 & 23 & 83 \\
\hline & C-Externalizing & 59.75 & 12.03 & 37 & 97 \\
\hline & C-CBCL Total & 58.39 & 11.00 & 37 & 91 \\
\hline \multirow{2}{*}{ Cognitive Competence } & C-ASQ Communication ${ }^{\mathrm{a}}$ & .91 & .65 & .53 & 3.87 \\
\hline & C-ASQ Problem Solving ${ }^{\mathrm{b}}$ & .76 & .34 & .42 & 1.67 \\
\hline
\end{tabular}

Note. M - Mother Variables, C - Child Variables.

a,b Proportional score: the cut-off scores divided by the child ${ }^{\circ} Ø$ s actual score of the domain. 
Table 2. Correlation among the Study Variables $(n=67)$

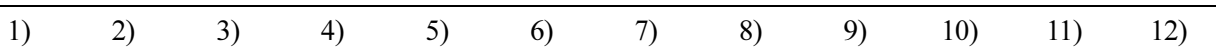

1) M-Sensitivity

2) M-Structuring $.78 * * * *$

3) M-Nonintrusive

$.63 * * * * .52 * * * *$

4) M-Non-hostility

$.62 * * * * \quad .53 * * * * \quad .55 * * * *$

5) C-Responsiveness

$.86 * * * * .74 * * * * \quad .58 * * * * \quad .59 * * * *$

6) C-Involvement

$.85 * * * * .75^{* * * *} .63 * * * * \quad .60 * * * * .93 * * * *$

7) M-Depress

$\begin{array}{llllll}-.24^{*} & -.23^{t} & -.41^{* *} & -.20 & -.27 * & -.29 *\end{array}$

8) M-Parental Stress

$\begin{array}{llllll}-.26^{*} & -.23^{t} & -.45^{* * *} & -.37 * * & -.31^{*} & -.39 * *\end{array}$

9) C-Internalizing

$\begin{array}{llllll}-.14 & -.11 & -.32 * * & -.26^{*} & -.21^{t} & -.24 *\end{array}$

10) C-Externalizing $\quad-.31 * \quad-.37 * * \quad-.34 * * \quad-.41^{* *} \quad-.36^{* *} \quad-.38^{* *}$

$.59 * * * *$

11) C-CBCL Total $\quad-.25^{*} \quad-.26^{*} \quad-.34 * * \quad-.41 * * \quad-.30^{*} \quad-.31 *$

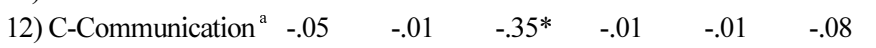

$.49 * * * * .64 * * * *$

$.53 * * * * .64 * * * * .62 * * * *$

$.56 * * * * .69 * * * * .89 * * * * .88 * * * *$

13) C-Problem Solving ${ }^{\mathrm{b}} \quad .03$

$\begin{array}{llllll}-.03 & -.24 & -.03 & -.04 & -.11\end{array}$

$.44 * * .28 * \quad .13$

$.28 * \quad 31 * \quad 35 * * \quad 24 *$

Note. ${ }^{* * * *} p<.0001, * * * p<.001,{ }^{* *} p<.01, * p<.05,{ }^{t} p<.10$.

$\mathrm{M}$ - Mother variables, $\mathrm{C}$ - Child variables

a, b ASQ refers to the proportional scores of the scale: the greater ASQ proportional score indicates the more risk of having a deficit in that domain. ' Communication addresses babbling, vocalizing, listening, and understanding. The score means the 'deficit in communication.' b Problem Solving subscale addresses child's learning abilities and skills of playing with toys. The score means the 'deficit in problem solving ability.'

provides evidence of the predictive value of these qualities). The data indicated that deficits in child communication and problem solving skills were associated with higher parenting stress, depression, and child behavior problems. Deficits in child communication correlated negatively with maternal non-intrusiveness where mothers of children who have difficulties in communication were more likely to be observed as being intrusive.

\section{Effects of Maternal Psychological Well-being and EA on Child Behavior Problems}

SEM was completed using maximum likelihood estimation available within AMOS software (version 17.0; Arbuckle, 2007) in order to examine the major hypotheses of this study. The SEM permits the examination of the direct and indirect relations among the major study variables. The model fit was assessed with commonly available fit indices, i.e., $\chi^{2}$ statistics, Comparative Fit Index (CFI; Bentler \& Bonett, 1980) and the root mean square error of approximation (RMSEA; Browne \& Cudeck, 1993). The $\chi^{2} / d f$ ratio between 1 and 3 indicates appropriate fit (Arbuckle \& Wothke, 1999), CFI value from .9 to .95 are considered indicative of good model fit and is more acceptable as it approaches the values of 1
(Bentler, 1990). Less error is more desirable and a RMSEA close to the value of 0 is considered a better fit. In general, the RMSEA <.08 indicates an acceptable fit (Browne \& Cudeck, 1993).

For the first research question, structural models were tested to determine whether EA mediates the association between maternal psychological wellbeing and child behavior problems. First, the model with a direct path coefficient from maternal psychological well being to child behavior problems was examined $(\mathrm{A} \rightarrow \mathrm{C})$. Model fit indices were indicated as $\chi^{2}(1)=.19, \mathrm{p}=.662 ; \chi^{2} / d f$ ratio $=.19$; $\mathrm{CFI}=$ 1.00, and RMSEA $=.000$ (90\% C.I.=.000-.248). Path coefficients directed from maternal psychological well-being to child behavior problems was $\beta=.87$. These values indicate that there is high probability that the model tested with the present data will have an exact fit with the population model. In addition, RMSEA being 0 indicates that the model has an exact fit; therefore, SEM analysis result indicated that there is a direct effect of maternal psychological wellbeing on child behavior problems.

As for the alternative models, the partial mediation and the full mediation of EA were examined (Figure 1). The partial mediation model included the paths from maternal psychological 


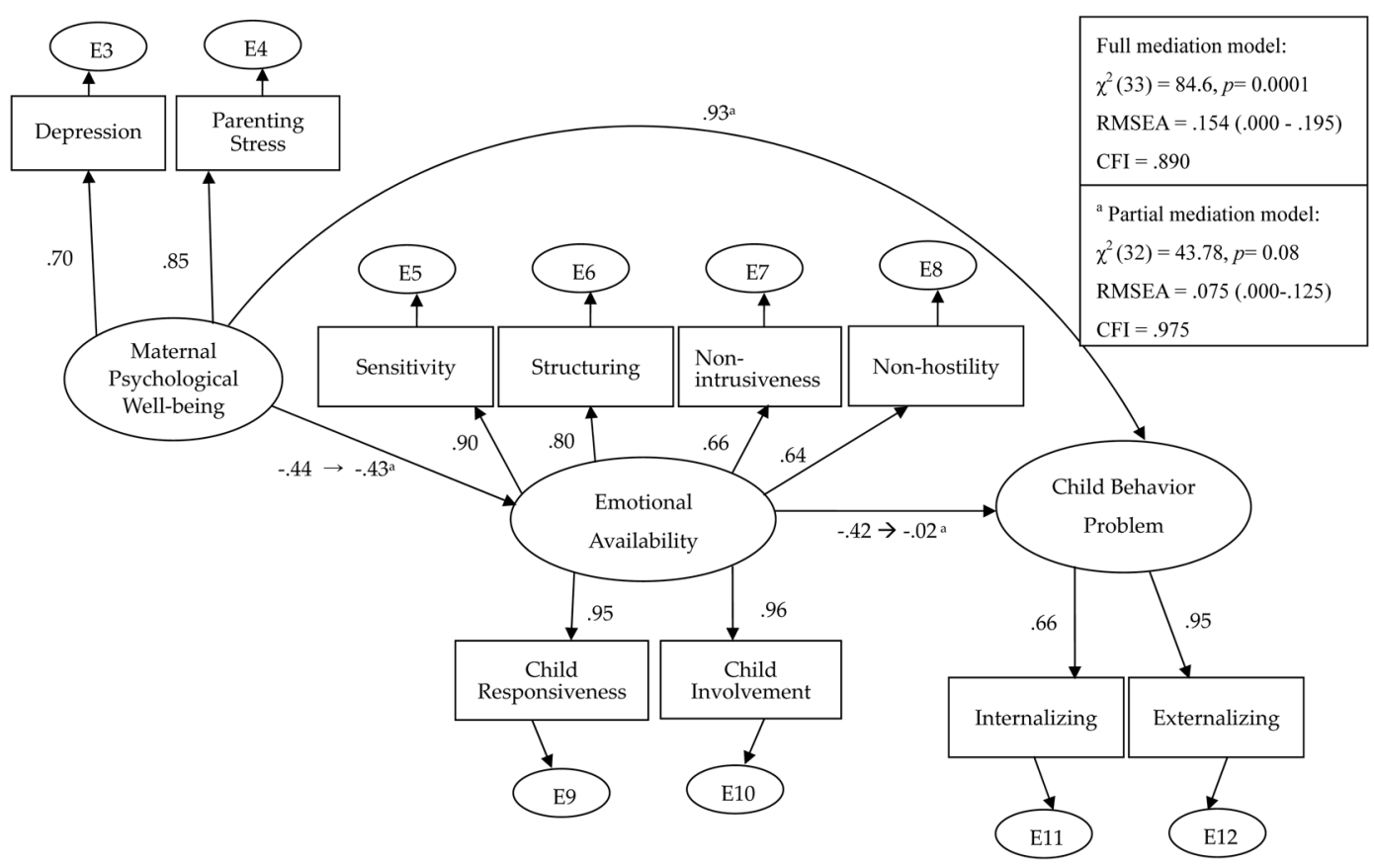

Figure 1. Structural Equation Modeling Predicting Child Behavior Problems ( $n=67)$

Note. The values on the arrows indicate the standardized beta weights.

Variables or arrows not significant are excluded.

${ }^{a}$ When direct effect from psychological well being to child behavior problems were added.

well-being to EA $(\mathrm{A} \rightarrow \mathrm{B})$, EA to child behavior problems $(\mathrm{B} \rightarrow \mathrm{C})$, and maternal psychological wellbeing to child behavior problems $(\mathrm{A} \rightarrow \mathrm{C})$. The full mediation model freed the direct path from maternal psychological well-being to the behavior problems $(\mathrm{A} \rightarrow \mathrm{C})$.

The model fit for the full mediation of EA revealed the following fit indices: $\chi^{2}(33)=84.6, \mathrm{p}=$ $.0001 ; \chi^{2} / d f$ ratio $=2.56 ; \mathrm{CFI}=.890$, and $\mathrm{RMSEA}=.154$ (90\% C.I.=.000-.195). Path coefficients directed from the maternal psychological well-being to EA was $\beta=$ -.44 , and from EA to behavior problems was $\beta=-.42$, with both classified as significant.

The model fit for the partial mediation of EA was also examined; this created an additional direct effect of psychological well-being on child behavior problems to the full mediation model. The model fit indices were: $\chi^{2}(32)=43.8, p=.08 ; \chi^{2} / d f$ ratio $=1.37$; $\mathrm{CFI}=.975$, and $\mathrm{RMSEA}=.075$ (90\% C.I. $=.000-.125)$ (see the values footnoted with ${ }^{\text {a }}$ in Figure 1). When compared with the full mediation model, the partially mediated model produced a modest improvement in fit indices. However, the path coefficient from EA to behavior problems was no longer significant $(\beta=-.02)$. Among the three tested models, the $\Delta \chi^{2}$ test show that the model with no mediating factor (direct effect from $\mathrm{A} \rightarrow \mathrm{C}$ only) provides a significantly better fit with the data than the full or partial mediation models.

In the structural model, the six EA dimensions were the indicators of the latent variable labeled as EA. In order to examine the role of specific EA dimensions, the regression analyses and SEM models were examined using individual EA scales as mediators. However, the stepwise regression analyses for testing the mediating effect (Baron \& Kenny, 1986) failed to prove the mediation effect of individual EA scales. The SEM models indicated a poorer fit when the model was tested with individual EA dimensions instead of using one latent variable with six indicators. SEM models were examined only with four maternal EA scores and only two child EA scores in separate models; however, the model fit decremented as to have poorer fit. 


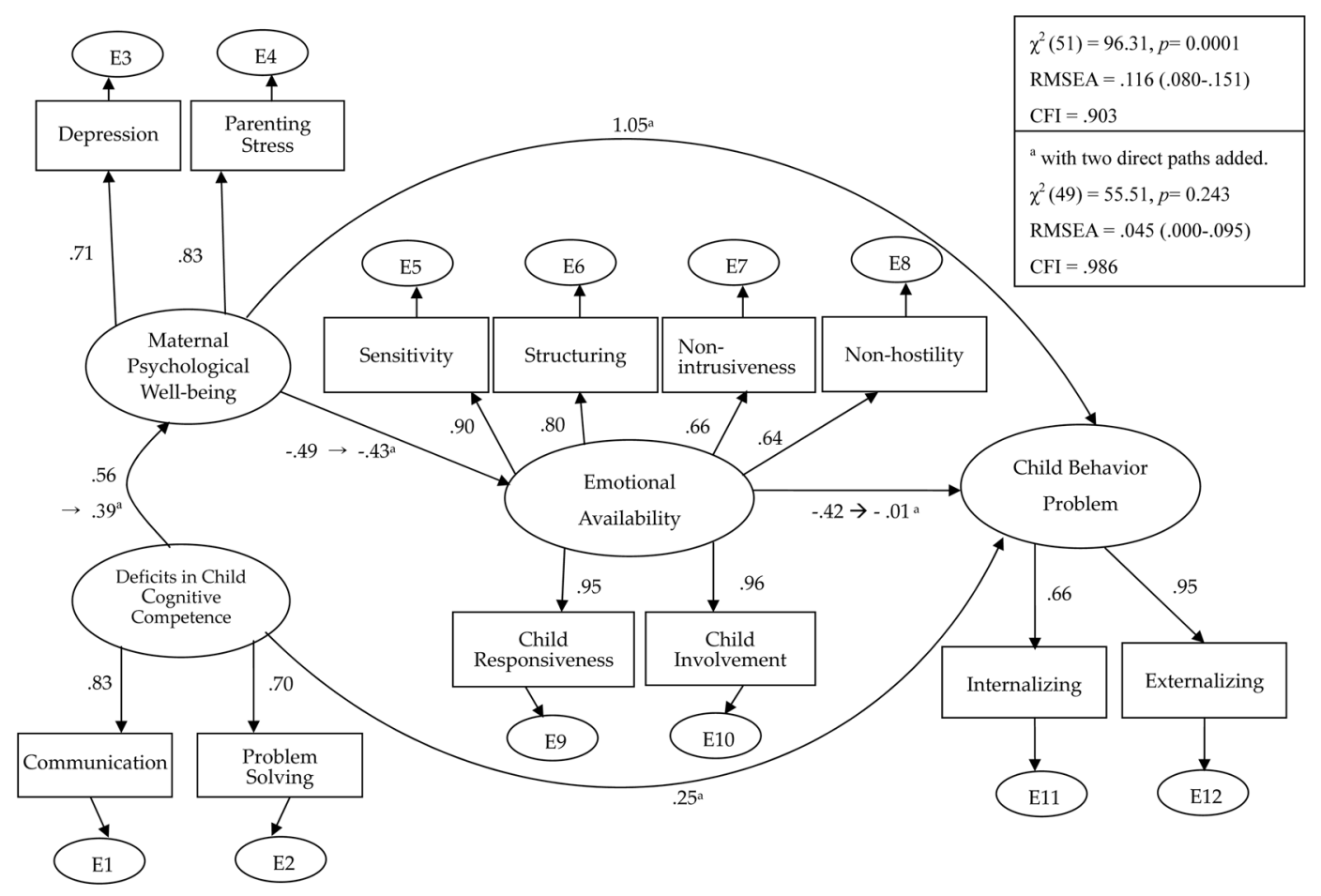

Figure 2. Structural Equation Modeling Predicting Child Behavior Problems ( $n=67)$

${ }^{a}$ When the two direct effect paths were added.

SEM analyses indicated that higher levels of maternal stress and depression were associated with lower EA and the lower EA was linked to more child behavior problems (at least in one model); however, maternal psychological well-being (maternal stress and depression) was found to have a direct and strong effect on child behavior problems. The models had a better fit when EA was used as a latent variable, rather than with the individual EA dimensions.

EA and Child Behavior Problems: Effects of Child

Cognitive Competence and Parental Psychological Well-being

A series of structural models examining the effects of child cognitive competence, parental psychological well-being, and EA on child behavior problems were analyzed to test the second hypothesis. The first model included three paths: the path coefficient from deficits in cognitive competence (A) to maternal psychological well being $(\mathrm{A} \rightarrow \mathrm{B})(\beta=.56)$, psychological well-being to EA $(\mathrm{B} \rightarrow \mathrm{C})(\beta=-.49)$ and EA to child behavior problems $(C \rightarrow D)(\beta=$ -.42) as shown in Figure 2. This model revealed the following fit indices: $\chi^{2}(51)=96.31, p=.00001, \chi^{2} / d f$ ratio $=1.88, \mathrm{CFI}=.903$, and $\mathrm{RMSEA}=.116$ (90\% C.I.= $.080-.151)$.

Two more direct coefficients were added, paths from deficits in cognitive competence to behavior problems $(\beta=.25)$ and from maternal psychological well being to behavior problems $(\beta=1.05)$ (see the values footnoted with ${ }^{a}$ in Figure 2). When these two additional paths were added, there was an improvement in the model fit that indicated a good model fit: $\chi^{2}(49)=55.51, p=.243 ; \chi^{2} / d f$ ratio $=1.13$, $\mathrm{CFI}=.986$, and RMSEA $=.045$ (90\% C.I.=.000-.095). The results indicate that more deficits in child cognitive competence are indirectly associated with lower mother-child EA and a lower mother-child EA is associated with more behavior problems. These results supported our second hypothesis, which suggested that a child's cognitive competence is indirectly associated to child behavior problems through the pathways of maternal psychological 
well-being and mother-child EA.

A series of structural models with individual EA dimensions were analyzed to test the role of specific EA dimensions. Separate models with only maternal EA dimensions and only child EA dimensions were tested as well. However, those models resulted in a poorer fit than the one with all EA dimensions that indicated one latent variable and only the final SEM model with best model fit was chosen to be reported.

\section{DISCUSSION}

\section{EA and Maternal Psychological Well-being}

One of the major objectives of this study was to explore the direct and indirect effect of maternal psychological well-being on child behavior problems. In this study, maternal depression and stress were used as the indicators of maternal psychological well-being. The indirect or meditational effect of EA was examined and the association between maternal psychological well-being and EA was examined as the first step of the preliminary analyses. Mothers with higher depressive symptoms and higher stress levels were shown to have lower maternal EA when interacting with their children. In addition, the children of mothers with high depression and stress displayed lower child EA (involvement and responsiveness) towards mothers. Thus, the present findings support the hypothesis that concurrent depressive symptoms and stress are related to mother-child EA.

The findings on maternal non-intrusiveness and maternal non-hostility are noteworthy. In order to look at the role of specific EA Scales, hierarchical regressions were analyzed individually with the specific EA variables used as dependent variables. (SEM models were also examined using the specific EA dimensions. However, the results reported that those model fits had a poorer fit than those using the 6 EA scales as a whole and the EA scales were used as a whole in this paper). The maternal nonintrusiveness scale appeared as a unique and important dimension of maternal EA associated with maternal depression and stress (maternal depressive symptoms and parenting stress together accounted for $30 \%$ of variance in maternal EA nonintrusiveness). Further, maternal non-hostility was strongly associated with maternal stress. These findings suggest that the non-intrusiveness and nonhostility of mothers are important EA dimensions in relation to maternal psychological well-being and children's functioning.

\section{Child Cognitive Competence, Maternal Psychological Well-being, and EA}

Findings from the SEM model indicate that deficits in a child's cognitive competence indirectly affect EA through maternal psychological well-being. Those children with more deficits in cognitive competence were more likely to have depressed and/or stressed mothers, and both depression and stress were correlated with EA. Deficits in child communication negatively correlated with maternal EA nonintrusiveness. Children who have better communication and problem solving skills may help their mothers be more effective in interaction.

\section{EA and Child Behavior Problems: Communication Skills and Parental Psychological Well-being}

Maternal EA and child EA were significantly and negatively associated with children's internalizing as well as externalizing problems. In support of these findings, Ginsburg, Grover, \& Ialongo's (2004) in their six-year follow-up study reported that higher levels of maternal criticism, intrusiveness, and overcontrolling behavior were significantly related to higher anxiety symptoms in children (seeking reassurance, expressing self-doubt, and/or expecting perfection from themselves). Further, Mills and Rubin (1993) also reported that mothers of socially withdrawn 4 4/2-year-old children displayed more directive teaching, coercive strategies, as well as angry behavior than mothers of children who were less so. In addition, Eckerman and Oehler (1992) explained that children tend to withdraw from mothers that over-stimulate him them. Children who withdraw from their mothers may generalize these early experiences, may become avoidant with new people, and develop a socially withdrawn style.

The correlation between maternal hostility and child externalizing problems can be explained 
through social learning theory as well (Bandura, 1989). If parents exhibit hostility as a strategy in conflict resolution, their children may acquire such unfavorable strategies by watching and listening to their parents. However, considering the dynamic nature of relationships, it is also possible that the children with externalizing problem behaviors might increase the frequency and intensity of maternal hostility.

The SEM findings did not support the first hypothesis that EA mediates the association between maternal psychological well-being and child behavior problems. The fit indices were not high enough to prove the close fit of the model; however, the correlation results indicated the potential mediating role of EA between the two variables. The SEM may have revealed different results if the model was examined with a larger sample size and the data were collected at different time points. In addition, the characteristics of this sample may have contributed to direct and strong effects of maternal psychological well-being on child behavior problems. In general, the participants were low-income families and $48 \%$ of the mothers had high total parenting stress scores $\left(\geq 85^{\text {th }}\right.$ percentile in the general population) and $35.8 \%$ of mothers were classified as clinically depressed (i.e., CES-D total $\geq 16$ ).

These findings support the well-known relation between maternal depression and a child's internalizing and externalizing problems (Downey \& Coyne, 1990; Leadbeater et al., 1996). Although there was some limitations in testing the direction of causality since the data were collected concurrently, we can also posit the alternative direction of the effects from the child to the mother. Mothers with children that have more behavior problems may be at higher risk of feeling more stressed and depressed. For example, Jackson (2000) argued that the most robust predictor of parenting stress is a child's behavior problems.

The findings of the current study suggest that in addition to maternal depression, parenting stress is predictive of child behavior problems. This may be because the actual items included in the assessment used for maternal stress are directly related to problems that concern parenting issues that includes the mother's perception of the child difficultness. For example, some of the items in the Parenting Stress Index-Short Form (PSI-SF, $3^{\text {rd }}$ ed.; Abidin, 1995) are "My child is so active that it exhausts me", "My child seems to be much harder to care for than most", "I have had many more problems raising children than I expected", and "My child gets upset easily over the smallest thing." A high correlation may be expected because both PSI-SF and CBCL are the mothers' self-reports on perceptions of their children.

The SEM analyses supported the second hypothesis that a child's cognitive competence has an indirect effect on a child's behavior problems through the pathways of maternal psychological well-being and EA. The greater the deficits in cognitive competence (especially communication skills) the more psychological problems reported by mothers. Maternal depression and stress were negatively related to mother-child EA that was linked to more child behavior problems. This finding suggests a role for a child's internal resources that may contribute to the EA with the caregiver that might eventually influence on child outcome (e.g., behavior problems).

SEM results revealed a closer fit when the EA was considered as one latent variable instead of running the model with individual EA dimensions. In addition, structural models were examined with internalizing and externalizing problems treated as independent indicators representing the child behavior problems; however, they did not improve the model fit. This may be because in SEM, a single observed variable to assess a latent variable increases the measurement error and the possibility of poorly defining the construct. Thus, in SEM, it is recommended to use multiple indicator variables for each latent dependent and independent variable (Schumacker \& Lomax, 2004). Therefore, there was limitation to examine the different roles of each EA dimension on child behavior problems through the model testing. However, correlation results provided information of which specific EA dimensions might have had greater relations to the problems faced by the mother and child. Maternal EA non-intrusiveness strongly related to maternal depression and stress was significantly associated with child 
communication skills and behavior problems. Maternal non-intrusiveness, non-hostility and child involvement were significantly associated with a child's internalizing problems; however, all of the mother-child EA dimensions were related to a child's externalizing problems. These findings highlight the need to consider specific aspects of EA and a child's characteristics in the prediction or prevention of a child's behavior problems.

The findings for the study suggest important practical applications for the growing field of infant mental health intervention. Practitioners working in the field of intervention, especially for those who work in early intervention (from birth to 3 years) often involve the primary caregiver and child as a paired target. For most of the early intervention programs, training the caregivers (mothers, teachers, and childcare providers) to be more sensitive and responsive in the interaction with the child is considered an important ingredient. EA with mutual negotiation in the interacting behavior may be a useful tool for the prevention and intervention of psychological problems in both parents and children.

\section{REFERENCES}

Abidin, R. R. (1995). The Parenting Stress Index (PSI, $3^{\text {rd }}$

Ed.). Odessa, FL: Psychological Assessment Resources. Achenbach, T. M., \& Rescorla, L. A. (2000). Manual for ASEBA preschool forms $\mathcal{E}$ profiles. Burlington, VT: University of Vermont, Research Center for Children, Youth, \& Families.

Ainsworth, M. D. S., Blehar, M.C., Waters, E., \& Wall, S. (1978). Patterns of attachment: A psychological study of the Strange Situation. Hillsdale, NJ: Erlbaum.

Alpern, L., \& Lyons-Ruth, K. (1993). Preschool children at social risk: Chronicity and timing of maternal depressive symptoms and behavior problems at school and at home. Developmental Psychopathology, 5, 371-387.

Arbuckle, J. L. (2007). AMOS 16.0 user's guide. Chicago, IL: SPSS Inc.

Arbuckle, J. L. \& Wothke, W. (1999). AMOS 4.0 user's guide. Chicago: SPSS.

Bandura, A. (1989). Social cognitive theory. In R. Vasta (Ed.), Annals of Child Development, Vol. 6, (pp. 1-60). Greenwich, CT: JAI Press.

Baron, R. M., \& Kenny, D. A. (1986). The moderatormediator variable distinction in social psychological research: Conceptual, strategic, and statistical considerations. Journal of Personality and Social Psychology, 51(6), 1173-1182.

Bentler, P. M. (1990). Comparative fit indexes in structural models. Psychological Bulletin, 107, 238-246.

Bentler, P. M., \& Bonett, D. G. (1980). Significance tests and goodness-of-fit in the analysis of covariance structures. Psychological Bulletin, 88, 588-606.

Biringen, Z., Robinson, J., \& Emde, R.N. (1998). The Emotional Availability Scales ( ${ }^{r d}$ Ed.). Unpublished manual. Colorado State University, Fort Collins, CO, USA.

Biringen, Z., Robinson, J., \& Emde, R. N. (2000). Appendix B: The Emotional Availability scales ( $3^{\text {rd }}$ Ed.; An abridged infancy/early childhood version). Attachment \& Human Development, 2, 256-270.

Bretherton, I. (2000). Emotional availability: an attachment perspective. Attachment and Human Development, 2(2), 233-241.

Bronfenbrenner, U. (1979). The ecology of human development: Experiments by nature and design. Cambridge, MA: Harvard University Press.

Browne, M. W., \& Cudeck, R. (1993). Alternative ways of assessing model fit. In K. A. Bollen \& J.S. Long (Eds.), Testing structural equation models (pp. 136162). Thousand Oaks, CA: Sage.

Crowell, J.A. \& Feldman, S. (1988). Mothers' internal models of relationships and children's behavioral and developmental status: A study of mother-child interaction. Child Development, 59, 1273-1285.

Dombrowski, S. C., Timmer, S. G., Blacker, D. M., \& Urquiza, A. J. (2005). A positive behavioural intervention for toddlers: Parent-child attunement therapy. Child Abuse Review, 14(2), 132-151.

Downey, G., \& Coyne, J.C. (1990). Children of depressed parents: An integrative review. Psychological Bulletin, 108(1), 50-76. 
Easterbrooks, M.A., Biesecker, G., \& Lyons-Ruth, K. (2000). Infancy predictors of emotional availability in middle childhood: the roles of attachment security and maternal depressive symptomatology. Attachment and Human Development, 2, 170-187.

Eckerman, C. O., \& Oehler, J. M. (1992). Very lowbirth weight newborns and parents as early social partners. In S. L. Friedman \& M. D. Sigman (Vol. Eds.), Annual advances in applied developmental psychology: Vol. 6. The psychological development of lowbirthweight children (pp. 91-124). Norwood, NJ: Ablex.

Emde, R. N. (2000). Next steps in emotional availability research. Attachment and Human Development, 2(2), 242-248.

Ginsburg, G. S., Grover, R. L., \& Ialongo, N. (2004). Parenting behaviors among anxious and non-anxious mothers: Relation with concurrent and longterm outcomes. Child $\mathcal{E}$ Family Behavior Therapy, $26(4), 23-41$

Harnish, J. D., Dodge, K. A., \& Valente, E. (1995). Mother-child interaction quality as a partial mediator of the roles of maternal depressive symptomatology and socioeconomic status in the development of child behavior problems. Child Development, 66(3), 739-753.

Jackson, A. P. (2000). Maternal self-efficacy and children's influence on stress and parenting among single black mothers in poverty. Journal of Family Issues, 21(1), 3-16.

Leadbeater, B. J., Bishop, S. J., \& Raver, C. C. (1996). Quality of mother-toddler interactions, maternal depressive symptoms, and behavior problems in preschoolers of adolescent mothers. Developmental Psychopathology, 32, 280-288.

Mahler, M. S., Pine, F., \& Bergman, A. (1975). The psychological birth of the human infant: Symbiosis and individuation. New York: Basic Books.
Mills, R. S. L., \& Rubin, K. H. (1993). Socialization factors in the development of social withdrawal. In $\mathrm{K}$. H. Rubin, \& J. B. Asendorpf (Eds.). Social withdrawal, inhibition and shyness in childhood (pp. 117150). Erlbaum: Hillsdale, NJ.

Moreno, A. J., Klute, M. M., \& Robinson, J. L. (2007). Relational and individual resources as predictors of empathy in early childhood. Social Development, 17(3), 613-637.

Radloff, J. S. (1977). The CES-D Scale: A self-report depression scale for research in the general population. Applied Psychological Measurement, 1, 385-401.

Schumacker, R. E., \& Lomax, R. G. (2004). A beginner's guide to structural equation modeling $\left(2^{\text {nd }}\right.$ Ed.). Mahwah, NJ: Lawrence Erlbaum Associates, Inc.

Shrout, P. E. \& Fleiss, J. L. (1979). Intraclass correlations: Uses in assessing rater reliability. Psychological Bulletin, 86(2), 420-428.

Squires, J. Potter, L., \& Bricker, D. (1999). The ASQ User's Guide for the Ages \& Stages Questionnaires: A parent-completed, child-monitoring system ( $2^{\text {nd }}$ ed.). Baltimore: Paul. H. Brookes Publishing.

van Doesum, K. T. M., Riksen-Walraven, J. M., Hosman, C. M. H., \& Hoefnagels, C. (2008). A randomized controlled trial of a home-visiting intervention aimed at preventing relationship problems in depressed mothers and their infants. Child Development, 79(3), 547-561.

Vygotsky, L.S. (1978). Mind in society: The development of higher mental processes. M. Cole, V. John-Stenier, S. Scribner \& E. Souberman (Eds.). Cambridge, MA: Harvard University Press.

Received September 30, 2011

Revised November 19, 2011

Accepted November 20, 2011 